\title{
Technical methods for accelerating digital transformation of Chinese enterprises
}

\author{
Xinjian Qiang* \\ School of Computer Science, Xi'an Shiyou University, Xi'an, China
}

\begin{abstract}
Chinese enterprises are facing the opportunity of digital transformation, and all enterprises are advancing in exploration. This paper introduces the trend and challenge of digital transformation of Chinese enterprises. This paper expounds the digital transformation strategy of enterprises as the center. This paper discusses the four development processes of enterprise digital transformation, including digital empowerment, process optimization, digital transformation and digital reengineering. This paper puts forward the methods that Chinese enterprises should adopt in the process of transformation. First of all, the digital transformation of enterprises should be scientifically planned. Then select pilot projects in enterprises and try them out first. After that, the trial effect was evaluated. After summarizing and improving, copy and enlarge the scope. Finally, optimize the operation of the enterprise, and constantly adjust.
\end{abstract}

\section{Introduction}

At present, the modern information technology represented by the Internet, big data, artificial intelligence and so on is changing with each passing day. A new round of scientific and technological revolution and industrial transformation is booming, which has had a significant and far-reaching impact on enterprises all over the world. It is the trend of the times and the world development to accelerate the digital industrialization and industrial digitization and promote the deep integration of the digital economy and the real economy [1].

China is in the key window period from a big network country to a network power, becoming a global leader in Internet development, and is also a period of transformation and upgrading of digital technology and economic society, and the digitalization of traditional industries. Through the digital transformation drive management promotion, the use of new technology and business model innovation, digital transformation from local planning and design to global planning and top-level design [2].

Chinese enterprises need to further enhance the sense of responsibility and sense of urgency to accelerate the digital transformation, accelerate the organizational innovation, technological innovation, integration innovation and cross-border innovation of enterprises, deeply promote the digital transformation work, promote the quality change, efficiency change and power change, improve the industrial basic ability and the modernization level

\footnotetext{
*Corresponding author: xasyu@126.com
} 
of the industrial chain, and cultivate the global competitiveness World class enterprises should speed up the construction of a new pattern of high-quality development [3].

As an important means to transform and enhance the traditional kinetic energy of enterprises and cultivate new kinetic energy of enterprises, digital transformation needs to deepen the understanding of the arduousness, long-term and systematic nature of digital transformation. Chinese enterprises need to play a leading role in the new round of scientific and technological revolution and industrial transformation, further strengthen the concept of digital transformation such as data-driven, integrated innovation and win-win cooperation, systematically organize the centralized learning of digital transformation theory, methods and practice, and actively carry out innovation competitions, achievements promotion, standard setting, exchange and training and other forms of activities to stimulate China Enterprise grass-roots vitality, to create courage, willing, good at digital transformation of the atmosphere.

\section{Challenges of digital transformation of Chinese enterprises}

From the perspective of the economic environment, the global digital economy is growing strongly, and the GDP of the United States digital economy accounts for more than $58 \%$. China's digital economy continues to develop rapidly, and the scale of China's digital economy continues to expand. Chinese enterprises have always attached great importance to information construction. Digital equipment is widely used in the production level. Information systems have covered the main business areas. The information construction and application of management have been widely covered. Mobile applications have been widely used. The sharing platform, procurement and sales e-commerce platform have achieved remarkable results. The construction of backbone network, data center and cloud platform has been formed Safe and reliable digital infrastructure has laid a solid technical foundation for digital transformation [4].

Enterprises are facing the problems of data governance, business standardization and the construction of digital talent team. At the strategic level, enterprises need to face three challenges.

One is the strategic ambition of the enterprise. Transformation is the development strategy of an enterprise, which can not be achieved overnight. It must be promoted by the senior managers of the strategic level, and needs the unified understanding, long-term planning and comprehensive top-level design of the strategic level. Enterprise IT leaders need to obtain the necessary recognition from the company's decision-making level and other business departments to negotiate and promote the required changes. Transformation is the enterprise revolution driven by data. It leaders must promote the top management to form a unified development cognition and strategic joint force in action. We should adopt the overall cooperation mode, such as the formation of strategic team, innovation round table, etc., to promote greater cooperation.

The second is the cultivation of digital culture. The success rate of digital transformation of large international companies is less than $10 \%$, and the key factor of failure is the lack of digital culture. The development speed of digital technology exceeds the development speed of any industry technology in the traditional sense. The more industry leading companies are, the more they focus on the professional technology of the industry, and the more profound professional culture atmosphere they have, the greater the resistance to the learning and acceptance of digital culture. It is very important to realize this point. Only by consciously cultivating new digital culture can the digital transformation be stable and successful.

The third is to adapt to the organizational change of digital transformation. Digital transformation is an advanced stage of enterprise information development. The 
construction and application of many information systems must have corresponding organizational guarantee to achieve the desired results. For example, we have built a cross process business optimization platform, but without a dedicated optimization team, users are still scattered in all aspects of the process, and the platform can not be put into operation normally. In fact, information construction has entered the deep water area. Informatization either leads to the change of organizational structure, or is bound by the traditional organizational structure and goes to failure.

\section{Human centered digital transformation strategy of Chinese enterprises}

It is very important to redefine the digital transformation strategy of enterprise organization based on human centered. In nature, people don't like complexity, but pursue simplicity, quickness and effectiveness. This is the core of enterprise digital transformation strategy research.

Digital technology is used to reconstruct the product, service, organization and business model of an enterprise. All industries need to rise to this height. But for specific industries, their entry points are different, and the pain points are different, so we need to reconstruct them according to the characteristics of the industry.

The starting point of digital transformation must start from the organizational strategy of the enterprise and the vision of the enterprise organization. Starting from solving the biggest pain point of the current enterprise, considering the biggest variable of today - the factor of digital transformation, it is necessary to use the way of digital transformation to solve the biggest pain point and the biggest strategic problem of enterprises today.

The digital transformation of enterprises is very complex, which needs new ideas, new methodology, new thinking, new technology and so on. Digital technology is always relatively simple. The realization of this digital transformation is organizational change. In fact, it is an interactive process among technology, organization and people, which requires new ideas and methodology.

\section{The process of digital transformation of Chinese enterprises}

With the development of digital technology, the digital transformation of enterprises is a new economic phenomenon integrating with business. This kind of integration should go through a progressive development path from nothing to existence, from low to high, from passive integration to active driving [5].

The first stage is digital empowerment. Digital empowerment is the primary stage and necessary stage of enterprise digital transformation. Through the practice of this stage, we can cultivate the digital culture of enterprises, improve the employees' digital consciousness and confidence in transformation. For example, through the use of digital technologies such as the Internet of things and mobile applications, the labor intensity of enterprise operation is greatly reduced, the number of employees is reduced, and the efficiency is improved, both enterprises and employees benefit. By enabling the product, we can quickly improve the product function and performance, improve the user experience, and increase the added value of the product. The characteristics of digital empowerment are small scale, low risk and quick effect.

The second stage is process optimization. Optimization is usually aimed at one or more business processes. On the basis of digitization, digital modeling technology is used to optimize the process. Optimization can be carried out in part of the process or in the whole process. Business process optimization usually requires high-level digitization, large 
amount of data accumulation, powerful modeling ability and huge computing power. In many cases, a large number of computers and instruments are needed to provide real-time data acquisition capabilities. Successful optimization projects usually have huge economic benefits.

The third stage is digital transformation. This is the original form of enterprise digital transformation. Through the empowerment of digital technology, the traditional business of enterprises can be easily transformed. Cloud computing is the most successful transformation of traditional computing power. It not only creates the world's first Amazon cloud, but also creates the entire cloud industry. Digital transformation is usually a complete business unit covering a certain range of enterprises. Because it has complete value, it is easier to "serve", and it is easier to find users and explore new markets.

The fourth stage is digital reconstruction. Reengineering is an advanced stage of digital transformation and a key step for traditional enterprises to transform into digital enterprises. Generally speaking, there are two types of Reengineering: one is the internal reorganization of production relations to adapt to digital productivity. It can be an independent business unit within the enterprise or an independent business unit of the whole enterprise. Through reengineering, the original enterprise will be rejuvenated and the digital productivity will be fully released. This kind of reorganization abandons the traditional organizational management structure, and the nature of the enterprise remains unchanged. Second, through cross-border M \& A, integration and innovation, we can break the boundaries of enterprises and realize business model reengineering. This kind of reengineering means gradually abandoning or changing the original core business and seeking a new profit model.

These four stages are not necessarily gradual, but can be developed alternately or in parallel. Especially in some large enterprises, different business units may be in different stages due to different business nature or digital level. The CEO of every enterprise, especially the CIO, should have a clear "digital transformation roadmap", accurately find the main direction of attack, and quickly realize the success of enterprise operation.

\section{Technical methods for digital transformation of Chinese enterprises}

The digital level of all walks of life is different, and the business characteristics and business environment are also very different. Therefore, it is impossible to have a uniform pace of digital transformation. To avoid the risk of transformation and maximize the value of transformation, we must follow the transformation implementation method of pilot first, step by step and step by step.

1. Scientific planning first: digital transformation has become a major trend in the development of Chinese enterprises. If we want to focus on the long-term and conform to the trend, we must plan a set of long-term development plan suitable for the characteristics of the industry and the current situation of the enterprise, so as to ensure the scientific and sustainable development of the enterprise.

2. Select pilot project: select the fields with mature conditions, talents, technology and other key elements as the starting point of the pilot project, so as to achieve quick results, accumulate experience and establish confidence.

3. Evaluation of pilot effect: evaluate the effect of the pilot work of enterprises at any time, and do not overestimate short-term benefits or underestimate long-term value. Timely find out problems, modify and improve the pilot measures quickly, and lay a solid foundation for the pilot work of digital transformation of enterprises.

4. Copy, expand and expand the scope: select similar or similar business scenarios, copy and enlarge the successful experience of pilot projects, and continuously expand the business and scene of enterprise digital transformation. 
5. Operation optimization, continuous adjustment: in the process of enterprise digital business operation, keep up with the pace of rapid development of new technology, and constantly optimize and quickly iterate. Evaluate the progress and success of digital transformation in time, and optimize and adjust the development plan of enterprise digital transformation. Circulation promotes the scientific, healthy and sustainable development of enterprises.

Enterprise digital transformation should follow the idea of five steps. Because digital transformation is a gradual exploration process of enterprise development, there are no mature and reliable cases in the use of digital technology or change of business model. Throughout the world's digital transformation cases, in all the transformation practices, the proportion of successful transformation cases that can achieve the expected goal is not high. In addition to the fuzzy concept of digital transformation and dislocation of evaluation system, there are many factors that need special attention. Clear concept, unified understanding and clear direction are the primary conditions for digital transformation. The fundamental guarantee of digital transformation is to enhance the strategic level of enterprises and the construction of digital technology capacity. The secret of successful transformation lies in the adoption of steady technological methods.

\section{Conclusion}

Digital economy is entering the fast lane in the global scope, and digitalization is becoming the new engine of Chinese enterprise strategy. Chinese enterprises must actively meet the digital era, take the initiative to transform to obtain sustainable and innovative enterprise development source power, and avoid being out of the big environment in the survival of the fittest. In the face of the international situation, the digital transformation of Chinese enterprises is imminent, and the digital transformation is a survival problem of enterprises. In terms of strategy, Chinese enterprises should follow the trend of global industrial interconnection, deconstruct and reconstruct the value chain of enterprise industry tactically, create a digital enabling platform for enterprise industry in terms of layout, and implement the ecological scheme for digital symbiosis of enterprise industry in the path. China's enterprises should combine with the actual situation, constantly strengthen digital awareness, build digital enterprises, and adopt feasible digital technology programs to promote the healthy and sustainable development of enterprises. Accelerating digital transformation is becoming the common goal of Chinese enterprises.

\section{References}

1. Zhu jie, The Journal of New Industrialozation, 9, 125-130 (2019)

2. Luca A. Finelli, Vas Narasimhan, Clinical Pharmacology \& Therapeutics, 108 (2020)

3. https://kns.cnki.net/kcms/detail/detail.aspx?doi=10.16619/j.cnki.rmltxsqy.2020.17.002.

4. Zhang Li, China's Foreign Trade, 9, 67-70(2020)

5. Li Jianfeng, Sinopec Monthly, 11, 24-25(2019) 